\title{
SPECT-CT Modality for Imaging of Medullary Thyroid Cancer (MTC)
}

Sonya Sergieva ${ }^{1^{*}}$, Mariana Atanasova ${ }^{2}$ and Ivan Terziev ${ }^{3}$

${ }^{1}$ Department of Nuclear Medicine, Sofia Cancer Center Sofia, Blvd, Bulgaria

${ }^{2}$ Department of Medical Oncology, Central Hospital, Plovdiv, Bulgaria

${ }^{3}$ Department of Pathology, UH" Queen Joanna", Sofia, Bulgaria

*Corresponding author: Sonya Sergieva, Department of Nuclear Medicine, Sofia Cancer Center, Sofia, 1784, Bulgaria. Tel: 35928752099; E-mail: Sergieva.sonya@yahoo.com

Received date: February 06, 2016; Accepted date: February 26, 2017; Published date: March 06, 2017

Copyright: @ 2017 Sergieva S, et al. This is an open-access article distributed under the terms of the Creative Commons Attribution License, which permits unrestricted use, distribution, and reproduction in any medium, provided the original author and source are credited.

\begin{abstract}
Medullary thyroid cancer (MTC) is a neuroendocrine tumor that arises from the parafollicular Calcitonin-producing C-cells of the thyroid. Typically the MTC is an extremely slow-growing cancer; however, it has a tendency of spreading distant metastases fairly early. Systemic chemotherapy and external been radiotherapy have not shown to give a good MTC response. As of date radical thyroidectomy is the main method of choice for therapy. New compounds like tyrosine kinase inhibitors (TKIs) targeting signaling pathways may have a positive outcome and be of great clinical benefits in patients with advanced and metastatic MTC. Somatostatin receptor are over expressed in MTC and thus allows the use of radiolabeled somatostatin analogues for scintigraphic imaging before and after treatment for proper staging and follow-up of these patients. SPECT-CT is used to optimize somatostatin-receptor scintigraphic protocols for MTC imaging. We have presented a case report of a patient who underwent total thyroidectomy with bilateral lymphadenectomy in August 2006 due to the diagnosed MTC. This patient was treated by chemotherapy and surgery during the period between January/2007- December/2014 because of the recurrent disease. In December 2014 the calcitonin level reached 56000 pg/ml; whole body scan with $740 \mathrm{MBq} 99 \mathrm{~m}$ TC-EDDA/HYNIC-TOC, followed by target SPECT-CT showed a total disease progression with advanced metastatic dissemination into the body. The assigned therapy was with Caprelsa ${ }^{\circledR}$ (Vandetanib) 300 $\mathrm{mg} / \mathrm{d}$ orally which was initialized from March 2015 until present. In June 2016 a control SPECT-CT somatostatinreceptor scintigraphy with $740 \mathrm{MBq} 99 \mathrm{~m}$ Tc-Tektrotyd was performed from which was reported a partial disease response with a reduction of about $60 \%$ in size and a decrease in the number of metastatic lesions shown to correlated with the decreased calcitonin level up to $1560 \mathrm{pg} / \mathrm{ml}$. It can be concluded that SPECT-CT with $99 \mathrm{mTC}$ Tektrotyd has important clinical role for re-staging and follow-up of patients with recurrent and metastatic MTC after target therapy.
\end{abstract}

Keywords: Medullary thyroid cancer; Target therapy; Caprelsa; SPECT-CT; ${ }^{99 \mathrm{~m}} \mathrm{Tc}-$ Tektrotyd

\section{Introduction}

Medullary thyroid cancer (MTC) is a neuroendocrine tumor that arises from the Para follicular Calcitonin-producing C-cells of the thyroid. MTC accounts for $5 \%$ to $10 \%$ of all thyroid malignancies [1]. It's been noted, that up to $75 \%$ of all MTC cases occur sporadically, and the rest $25 \%$ of the MTC are hereditary. Among the main prognostic factors for adverse outcome are; reduced calcitonin doubling time, advanced age at the time of diagnosis, the extent of the primary tumor, nodal disease and distant metastases [2]. Typically the MTC is an extremely slow-growing cancer; however it has a tendency of spreading distant metastases fairly early. The most common places have being to the region of the head and neck, as well as the chest cavity-primarily in the form of lymph nodes, followed by the bones, almost exclusively to the ribs, spinal vertebrae and pelvis, and finally to the liver and lung parenchyma $[1,2]$. Overall, loco-regional metastatic lymph nodes and distant secondary lesions in patients with MTC are reported in tumors with size under $1 \mathrm{~cm}[1,2]$. So far systemic chemotherapy and external been radiotherapy have not shown to give a good MTC response [1,3]. Therefore, early diagnosis and correct N/M-staging are of extreme importance for the management of MTC. As of date radical thyroidectomy is the main method of choice for therapy $[1,2]$. The treatment for sporadic and hereditary MTC patients without assessed lymph node metastases by a physical examination and cervical ultrasound is a total thyroidectomy with a bilateral prophylactic central lymph-node dissection (level V; IV), [1,2]. Lateral neck dissection (levels IIA, III, IV, V) is applied to patients with positive preoperative imaging [1,2]. Replacement thyroxine therapy is given to patients who have gone under a total thyroidectomy has been performed in order to maintain the serum TSH concentration within the normal range $[1,2]$.

C-cells are known for not producing thyroid hormone nor do they take up iodine. This is exactly why MTC is not treated with radioactive iodine. While malignant transformed $\mathrm{C}$-cells produce and secrete large amounts of peptides, including calcitonin, with few exceptions, when elevated serum calcitonin is spotted it is a sign for the presence of re-occurring MTC metastases after surgery. All patients with a serum CT concentration of $150 \mathrm{pg} / \mathrm{ml}$ should be screened for the presence of distant metastases [1,2].

As of the moment, there is no proven effective therapy for patients with advanced MTC [3]. New compounds like tyrosine kinase inhibitors (TKIs) targeting signaling pathways essential for tumor cell survival have been tested on patients with MTC metastases, that may have a positive outcome and be of great clinical benefits [2,3]. The new TKIs that are being tested in clinical trials include motesanib 
diphosphate, vandetanib, sorafenib and sunitinib show a partial responses from $2 \%$ to $35 \%$ and in the case of stabled disease the rates are from $27 \%$ to $87 \%$ with tolerable and manageable toxicity as differentiated thyroid cancer patients [2,3]. MTC tumors that are found to be positive for RET proto-oncogene mutation are more likely to be aggressive-therefore harder to treat and more likely to recur. Vandetanib has been approved by the United States Food and Drug Administration and the European Medicines Agency for the treatment of patients with locally advanced/metastatic MTC and should be considered for patients with incurable disease in which median progression free survival (mPFS) period is 30.5 months [1-3].

Vandetanib, a once-daily oral inhibitor of RET (Rearranged during Transfection) kinase, vascular endothelial growth factor receptor (VEGFR), and epidermal growth factor receptor signaling (EGFR), has previously shown antitumor activity in a phase II study of patients with advanced hereditary MTC [3].

Somatostatin receptors are over expressed in MTC and thus allows the use of radiolabeled somatostatin analogues for scintigraphic imaging before and after treatment for proper staging and follow-up of these patients $[4,5]$. Recently, SPECT-CT $\gamma$-cameras have become widely available which allows the fusion of anatomical CT and functional SPECT modalities [6,7]. SPECT-CT is used to optimize somatostatin-receptor scintigraphic protocols for MTC imaging.

\section{Case report}

A 68 year old man (J.A.V.) underwent total thyroidectomy with bilateral lymphadenectomy in August 2006 after clinical and ultrasound exams showing 4 thyroid nodules and left cervical lymphadenopathy. The diagnosis of MTC was made preoperatively by fine-needle aspiration biopsy. Pathological calcitonin immunohistochemical staining was positive for medullary thyroid cancer with left laterocervical lymph node metastases and normal appearing lymph nodes on the right side of the neck. The patient is without any underlying diseases and he has not been genetically tested. He was prescribed an L-Thyrox substitution therapy with $125 \mu \mathrm{g} /$ day to $150 \mu \mathrm{g} /$ day until January 2007 when the measured level of serum calcitonin was $817 \mathrm{pg} / \mathrm{ml}$. At that time six courses of chemotherapy with Epirubicin and Cisplatin were performed. The disease remained stabilized until December 2009 when the serum calcitonin level picked to $1880 \mathrm{pg} / \mathrm{ml}$. Clinical exam and ultrasound showed local recurrence and bilateral lymphadenopathy which were both removed surgically. Increased levels of serum calcitonin was noted in May $2012-1510 \mathrm{pg} / \mathrm{ml}$ when local recidives were found on the CT scan as well as left laterocervical lymphadenopathy and the spread of the disease- extending to both shoulder joints, accompanied with mediastinal and paratracheal lymph nodes. At this point, left selective laterocervical lymphadenectomy was performed followed by palliative radiotherapy. In December 2014 the calcitonin level was $56000 \mathrm{pg} / \mathrm{ml}$; whole body scan with $740 \mathrm{MBq}{ }^{99 \mathrm{~m} T c-E D D A} / \mathrm{HYNIC}-$ TOC ( ${ }^{99 \mathrm{~m}} \mathrm{Tc}$-Tektrotyd, Polatom) was performed, followed by target SPECT-CT (Symbia T2, Siemens) in the regions of the neck, upper thorax and abdomen (Figure 1). From the total body scan was derived a total disease progression with advanced metastatic dissemination into the thyroid bed, laterocervical area on both sides, left paratracheal, mediastinal and supraclavicular bilateral enlarged lymph nodes, bone metastasis in bilateral shoulder joints, pelvis, in retroperitoneal region with infiltration of right kidney, subcutaneous lesions and liver metastases we seen. The assigned therapy was with Caprelsa (Vandetanib) $300 \mathrm{mg} / \mathrm{d}$ orally which was initialized from March 2015 until present. In June 2016 a Control SPECT-CT somatostatin-receptor scintigraphy with $740 \mathrm{MBq}{ }^{99 \mathrm{~m}} \mathrm{Tc}$-Tektrotyd was performed (Figures 2) from which was reported a partial disease response with a reduction of about $60 \%$ in size and a decrease in the number of metastatic lesions shown to correlated with the decreased calcitonin level up to 1560 $\mathrm{pg} / \mathrm{ml}$. The ECOG performance status of the patient was 0 , and toxicity of the skin was demonstrated as grade 1 rash.
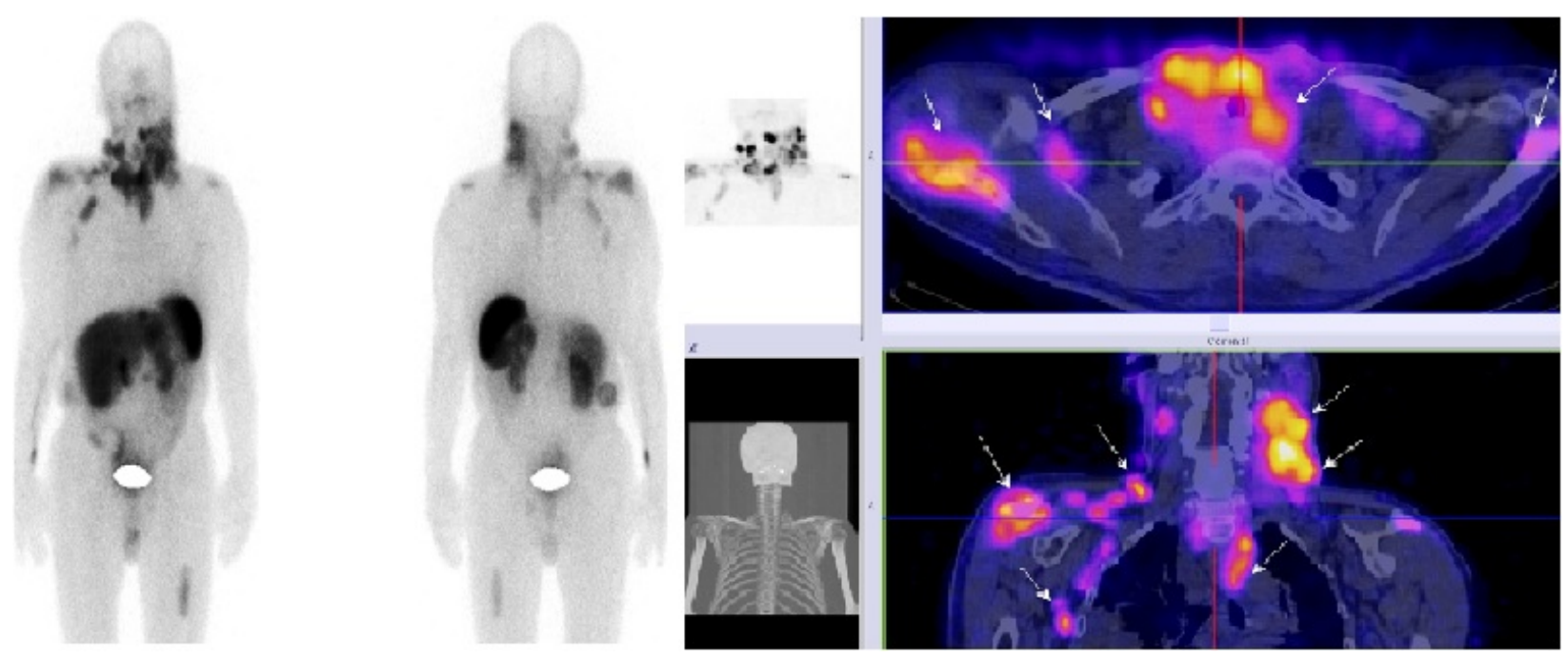

Figure1: M/68year-old with MTC after total thyroidectomy and cervical lymph node dissection- pT3pN1aM0. Disease recurrence with Calcitonin-56000 pg/ml in December 2014. Whole-body scan followed by SPECT-CT with 99m-Tektrotyd showed exact topographic disease extension in the region of the thyroid bed, bilateral laterocervical, left paratracheal, mediastinal and supraclavicular bilateral enlarged lymph nodes, bone metastasis in bilateral shoulder joints, left femur, in retroperitoneal region with infiltration of right kidney, subcutaneous lesions, liver metastases were imaged with high tracer uptake. 

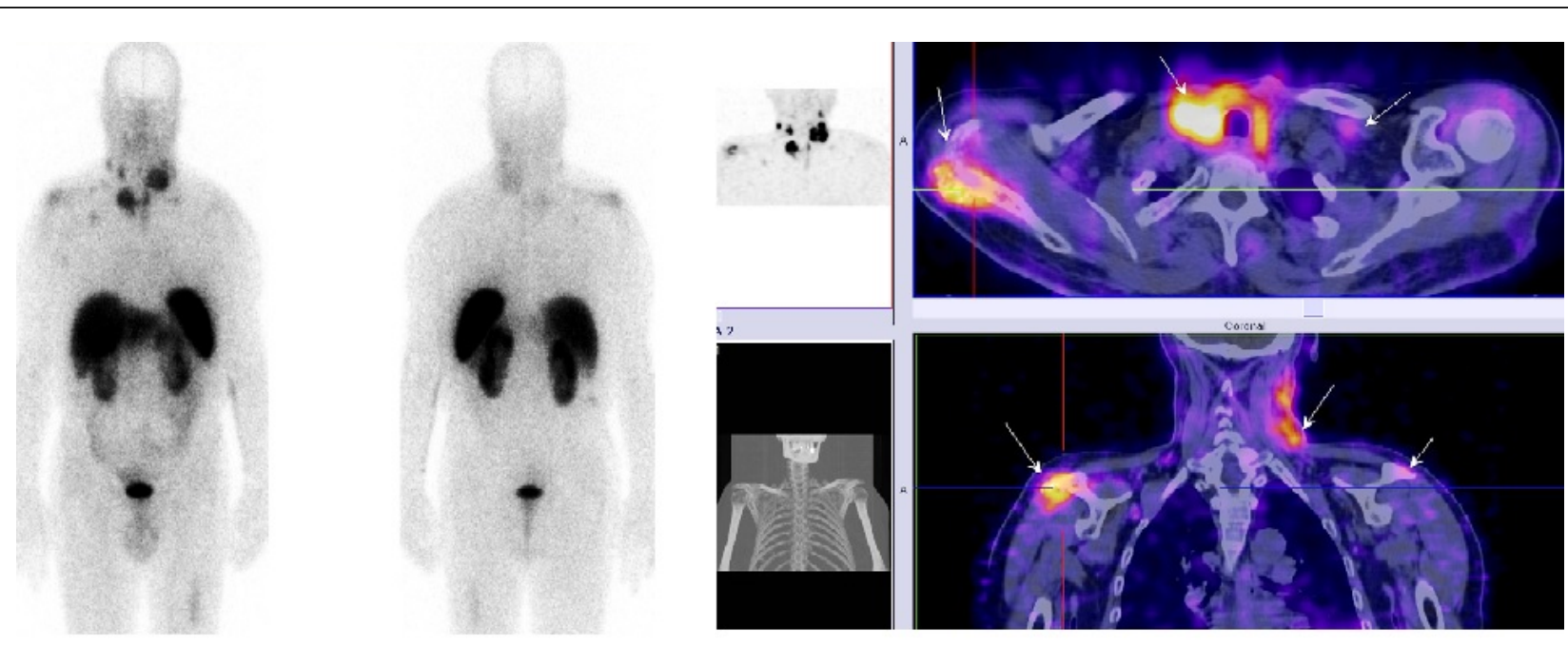

Figure 2: The same patient after target therapy with CAPRELSA/VANDETANIB/serum Calcitonin-1560 pg/ml in June 2016. Control wholebody scan followed by SPECT-CT showed reduced number and size of 60\% of metastatic lesions-partial therapeutic response.

\section{Discussion}

SPECT-CT somatostatin receptor imaging with radiolabeled somatostatin analogues has been recommended for staging and followup of patients neuroendocrine tumors $[5,8] .{ }^{99 \mathrm{~m}} \mathrm{Tc}$-Tektrotyd has high affinity to somatostatin receptors SSTR2 and lower to SSTR3 and SSTR5. Optimal physical parameters and biodistribution of ${ }^{99 \mathrm{~m}} \mathrm{Tc}-$ labelled somatostatin analog, lower background activity in the liver and bowel and imaging protocol performed 2 to 4 hours post injection on the same day are its priorities over ${ }^{111}$ In-pentetreotide [5,9].

Fusion SPECT-CT images improve image quality, show correct topography and morphological characteristics of metastatic lesions and provide exact differential diagnosis of the physiological from abnormal uncertain "hot" spots, thus increasing diagnostic accuracy of somatostatin-receptor SPECT studies [6-8]. Radionuclide targeting of somatostatin receptors for internal radiation therapy provides another therapeutic approach in advanced symptomatic non-operable MTC $[8,10]$.

Theranostics (therapy and diagnosis) using radiolabeled somatostatin analogues has proved to be a treatment method of choice in the individual management of neuroendocrine tumors overexpressed SSTR $[8,11]$. Peptide receptor radionuclide therapy (PRRT) using ${ }^{177} \mathrm{Lu}$-labeled or ${ }^{90}$ Y-labeled somatostatin analogues may have a significant clinical application in the management of MTC expressed SSTR established by SPECT-CT with ${ }^{111}$ In-pentetreotide/ ${ }^{99 \mathrm{~m}} \mathrm{Tc}$-Tektrotyd or PET-CT with ${ }^{68} \mathrm{Ga}$-labeled somatostatin analogues $[11,12]$.

Some authors have published the data of ${ }^{18} \mathrm{FDG}$ PET-CT in detection rate for imaging of recurrent MTC. They reported that sensitivity of ${ }^{18} \mathrm{FDG}$ PET-CT is $75 \%$ in patients with the calcitonin level above $1000 \mathrm{pg} / \mathrm{ml}$ and 20 to $36.8 \%$ when the calcitonin level is $500-1000 \mathrm{pg} / \mathrm{ml}$ or below $500 \mathrm{pg} / \mathrm{ml} .{ }^{18} \mathrm{FDG}$ PET-CT has may be of value to evaluate response to applied therapy in cases with progressive disease but there is no diagnostic information for the individual somatostatin-receptor status if PRRT is considered [13].

\section{Conclusion}

It can be concluded that SPECT-CT with ${ }^{99 m}$ Tc-Tektrotyd has important clinical role for staging and follow-up of patients with recurrent and metastatic MTC. The additional application of SPECTCT somatostatin-receptor scintigraphy in cases with MTC is to prove SSTR expression status in order to predict an individual therapeutic management including PRRT.

\section{References}

1. Nikiforov YE, Steward DL, Robinson-Smith TM, Haugen BR, Klopper JP, et al. (2009) Molecular testing for mutations in improving the fine-needle aspiration diagnosis of thyroid nodules. J Clin Endocrinol Metab 94: 2092-2098.

2. Cantara S, Capezzone M, Marchisotta S, Capuano S, Busonero G, et al. (2010) Impact of proto-oncogene mutation detection in cytological specimens from thyroid nodules improves the diagnostic accuracy of cytology. J Clin Endocrinol Metab 95: 1365-1369.

3. Brilli L, Pacini F (2011) Targeted therapy in refractory thyroid cancer: Current achievements and limitations. Future Oncol 7: 657-668.

4. Reubi JC, Caser B (2003) Concomitant expression of several peptide receptors in neuendocrine tumors: molecular basis for in vivo multireceptor tumour targeting. Eur J Nucl Med Mol Imaging 30: 781-79.

5. Parisella MG, Chianelli M, Alessandria CD,Todino V, Mikolajczak R, et al. (2012) Clinical indications to the use of 99mTc-EDDA/HYNIC-TOC to detect somatostatin receptor-positive neuroendocrine tumors. Q J Nucl Med Mol Imaging 56: 90-98.

6. Haslerud T (2014) SPECT-CT in neuroendocrine tumors. In: Clinical Application of SPECT-CT. Ahmadzadehfar H, Biersack HJ(eds). Springer-Verlag Heidelberg, New York, London 87-110.

7. Wong KK, Fig LM, Youssef E, Ferretti A, Rubello D, et al. (2014) Endocrine scintigraphy with hybrid SPECT/CT. Endocr Rev 35: 717-746.

8. Sergieva S, Robev B, Dimcheva M, Fakirova A, Hristoskova R (2016) Clinical application of SPECT-CT with 99mTc-Tektrotyd in bronchial and thymic neuroendocrine tumors (NETs). Nucl Med Rev Cent East Eur 19: 81-87.

9. Brabander T, Kwekkeboom DJ, Feelders RA, Brouwers AH, Teunissen JJM (2015) Nuclear Medicine Imaging of Neuroendocrine Tumors. In: Neuroendocrine tumors: A Multidisciplinary Approach. Papotti M, de Herder WW (eds). Front Horm Res. Basel, Karger 44: 73-87. 
Citation: Sergieva S, Atanasova M, Terziev I (2017) SPECT-CT Modality for Imaging of Medullary Thyroid Cancer (MTC). J Nucl Med Radiat Ther 8: 329. doi:10.4172/2155-9619.1000329

Page 4 of 4

10. Krenning EP, Kooij PP, Bakker WH (1994) Radiotherapy with a radiolabeled somatostatin analogue, [111In-DTPA-D-Phe1]-octreotide. A case history. Ann N Y Acad Sci 733: 496-506.

11. Salavati A, Puranik A, Kulkarni HR, Budiawan H, Baum RP (2016) Peptide Receptor Radionuclide Therapy (PRRT) of medullary and nonmedullary thyroid cancer using radiolabeled somatostatin analogues. Semin Nucl Med 46: 215-224.
12. Makis W, McCann K, McEwan AJ (2015) Medullary Thyroid Carcinoma (MTC) treated with 177Lu-DOTATATE PRRT: A report of two cases. Clin Nucl Med: 40: 408-412.

13. Skoura E, Datseris IE, Rondogianni P, Tsagarakis S, Tzanela M, et al (2012) Correlation between calcitonin levels and [18F]FDG PET/CT in the detection of recurrence in patients with sporadic and hereditary medullary thyroid cancer. ISRN Endocrinol 2012: 375231. 